\title{
Acute respiratory distress syndrome caused by carbon monoxide poisoning and inhalation injury recovered after extracorporeal membrane oxygenation along with direct hemoperfusion with polymyxin B-immobilized fiber column: a case report
}

Ji Hoon Jang ${ }^{1}$, Hang Jea Jang ${ }^{1}$, Hyun-Kuk Kim', Jin Han Park' ${ }^{1}$ Hyo-Jung Kim¹, Kyeong Min Jo', Woon Heo², Se Hun Kim ${ }^{3}$, Tae-Hoon $\mathrm{No}^{4}$ and Jae Ha Lee ${ }^{1 *}$

\begin{abstract}
Background: Inhalation injury from smoke or chemical products and carbon monoxide poisoning are major causes of death in burn patients from fire accidents. Respiratory tract injuries from inhalation injury and carbon monoxide poisoning can lead to acute respiratory distress syndrome and cytokine storm syndrome. In the case of acute respiratory failure needing mechanical ventilation accompanied by cytokine storm, mortality is high and immediate adequate treatment at the emergency department is very important.
\end{abstract}

Case presentation: This report describes a case of acute respiratory distress syndrome and cytokine storm followed by carbon monoxide poisoning in a 34-year-old Korean male patient who was in a house fire, and was successfully treated by extracorporeal membrane oxygenation and direct hemoperfusion with polymyxin B-immobilized fiber column at emergency department.

Conclusions: To prevent mortality in acute respiratory distress syndrome with cytokine storm from inhalation injury and to promote a better prognosis, we suggest that early implication of extracorporeal membranous oxygenation along with direct hemoperfusion with polymyxin B-immobilized fiber column even at the emergency department should be considered.

Keywords: Carbon monoxide poisoning, Smoke inhalation injury, Acute respiratory distress syndrome

*Correspondence: anilleus@naver.com

${ }^{1}$ Department of Internal Medicine, Haeundae Paik Hospital, Inje University College of Medicine, Busan 135-710, Korea

Full list of author information is available at the end of the article

\section{Background}

Inhalation injury is considered an important cause of morbidity and mortality in patients with burns from fire accidents. Direct thermal injury in the upper airway, smoke-induced chemical irritation in the lower airway, and systematic chemical or metabolic injury with various chemicals constitute the major pathophysiological background that can lead to severe acute respiratory distress 
syndrome (ARDS) [1]. Carbon monoxide (CO) poisoning is a leading cause of death among poisoning deaths worldwide and is most often caused by house fires. In the case of toxic concentration, increases in carboxyhemoglobin $(\mathrm{COHb})$ interfere with gas exchange in respiratory system, resulting in hypoxemia [2]. Hypoxemia from CO poisoning eventually causes tissue damage, which triggers an inflammatory response [3]. In terms of severe ARDS after inhalation injury, mortality is very high and aggressive treatment is needed in addition to conventional therapy including fluid restriction, protective ventilation, prone positioning, or nitric oxide inhalation [4]. Systemic inflammation caused by a "cytokine storm" due to excessive circulating cytokines also requires attention. Here, we present a case of $\mathrm{CO}$ intoxication and inhalation injury with secondary ARDS and cytokine storm in a patient who recovered after treatment with extracorporeal membrane oxygenation (ECMO) and direct hemoperfusion with polymyxin B-immobilized fiber (PMX-DHP) at the emergency department (ED).

\section{Case presentation}

A 34-year-old Korean male with no underlying diseases was rescued from a fire in his home. When the firefighters arrived, he was lying in his bed unconscious and pulseless. Ventricular fibrillation was observed on an automated external defibrillator monitor, and 2 minutes of chest compression with 150 J shock was applied. Recovery of spontaneous circulation ensued just after defibrillation, and the patient was transferred to the emergency department (ED). Upon arrival, erythema with vesicles was found in his face, neck, chest wall, both axillary areas, and the back. In addition, he had a low estimated Glasgow Coma Scale score (3 points) and weak respiration, so intubation and mechanical ventilation support were applied. The initial blood pressure was $88 / 46 \mathrm{mmHg}$. Laboratory tests showed leukocytosis, elevated hepatic enzymes (aspartate transaminase $310 \mathrm{U} / \mathrm{L}$, alanine transaminase $184 \mathrm{U} / \mathrm{L}$ ) and relatively normal levels of C-reactive protein $(0.07 \mathrm{mg} / \mathrm{dL})$ and procalcitonin $(0.07 \mathrm{ng} / \mathrm{dL})$. On the other hand, the level of serum interleukin 6 (IL-6) was markedly increased to $1328 \mathrm{pg} / \mathrm{mL}$. The initial arterial blood gas (ABG) showed hypoxemia with a low partial pressure of oxygen $\left(\mathrm{PaO}_{2} 37 \mathrm{mmHg}\right)$ as well as metabolic acidosis with a serum lactate level of $12.6 \mathrm{mmol} / \mathrm{L}$. The percentage of serum $\mathrm{COHb}$ was $59 \%$, indicating severe $\mathrm{CO}$ poisoning. An initial chest X-ray showed diffuse bilateral infiltration, and chest computed tomography $(\mathrm{CT})$ revealed diffuse ground-glass attenuation with an anterior-posterior density gradient by dense consolidation in dependent areas, which suggests an early state of ARDS (Figure 1). Therapeutic hypothermia was applied for prevention of hypoxic brain damage. Shock requiring infusion of norepinephrine exceeding the rate of $0.5 \mu \mathrm{g} / \mathrm{kg} /$ minute despite adequate fluid resuscitation was observed after sedation along with therapeutic hypothermia. Even with $100 \%$ of fraction of inspired oxygen $\left(\mathrm{FiO}_{2}\right)$ and maximal positive end expiratory pressure, hypoxemia $\left(\mathrm{PaO}_{2} 61 \mathrm{mmHg}\right)$ as well as hypercapnia (partial pressure of carbon dioxide $58 \mathrm{mmHg}$ ) progressed. Prone position ventilation and application of inhaled nitric oxide were considered, but were not implemented due to concern for rapid progression of shock. Therefore, we decided to conduct veno-veno extracorporeal
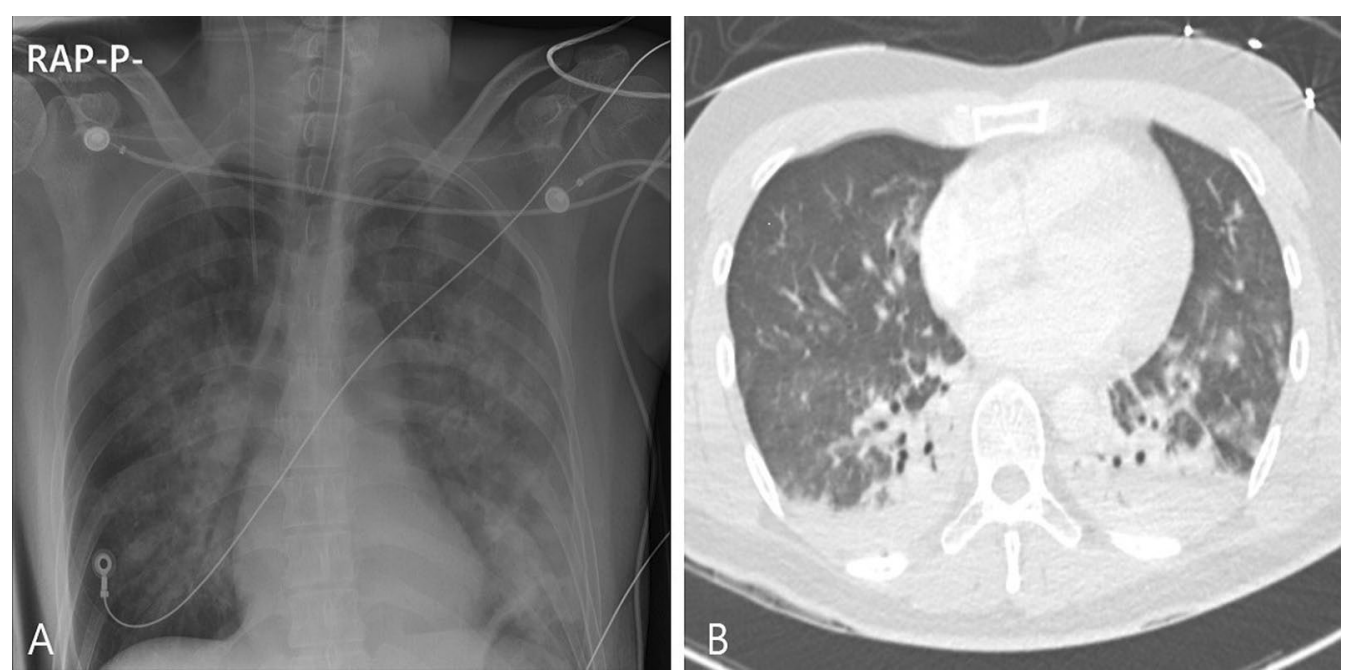

Fig. 1 Chest imaging findings. A Bilateral infiltrates on chest X-ray. B Anterior-posterior density gradient by consolidation in bilateral dependent area with background diffuse ground-glass opacities 
membranous oxygenator (V-V ECMO) via both right and left femoral venous cannulation. Despite V-V ECMO application, hypoxemia and shock progressed. Thus, direct hemoperfusion with polymyxin B-immobilized fiber (PMX-DHP) was indicated and conducted immediately to prevent further development of cytokine storm. PMX-DHP (TORAYMYXIN ${ }^{\mathrm{TM}}$ PMX-20R, TorayMedical, Tokyo, Japan) was applied with continuous renal replacement therapy (CRRT) via a nontunneled, doublelumen catheter inserted in left jugular vein, with a starting blood flow rate of $150 \mathrm{~mL} /$ minute for 24 hours. The patient was transferred to the intensive care unit (ICU) with ECMO and PMX-DHP. In the ICU on post-burn day 1 , flexible bronchoscopy was done to assess degree of inhalation injury and to toilet the airway. Bronchoscopy revealed severe edema and congestion in the bronchial wall with carbon soot deposition. (Figure 2) With $\mathrm{V}-\mathrm{V}$ ECMO maintenance and 24 hours of PMX-DHP, serum $\mathrm{COHb}$ percentage and IL-6 level normalized, and dramatic improvement on chest X-ray was seen within 96 hours. (Figure 3) On the seventh and eighth ICU days, the patient was weaned from V-V ECMO and the ventilator, respectively. On the 11th ICU day, the patient was moved to the general ward with a plan for systematic rehabilitation including respiratory rehabilitation. Upon transfer, the patient was alert without cognitive dysfunction, and electroencephalogram along with diffusion magnetic resonance imaging showed no definitive signs of hypoxic brain damage. However, both sensory impairment and motor weakness were observed in the right lower extremity with an abnormal result on electromyography suggesting right lumbar plexopathy. The patient was discharged from hospital with the plan of further rehabilitation through the outpatient clinic in the rehabilitation medicine department of hospital in other city.

\section{Discussion}

Inhalation injury is a disastrous injury that occurs in one-third of all burn patients, and smoke inhalation injury contributes to increased mortality, up to 20 times higher than that of burn alone $[5,6]$. Smoke inhalation injury can lead to not only long-term pulmonary dysfunction but also prompt respiratory complications such as pneumonia and acute respiratory distress syndrome. ARDS might be caused by contributing factors, including smoke toxicity, bronchorrhea, alveolar permeability, and inflammatory mediator expression. The incidence of ARDS in patients with burn and inhalation injuries who required mechanical ventilation was reported in the range of 34-43\% [7]. In terms of ARDS, mortality is up to $40 \%$; and intubation, fiberoptic bronchoscopy and mechanical ventilator support are currently used as initial treatments at the ED [8]. However, in cases of refractory, severe ARDS with maximal support of mechanical ventilation, ECMO should be considered as an additional option. Recently Dabras et al. suggested that ECMO is a viable therapeutic option and can contribute to improved survival rates in patients with ECMO for ARDS after inhalation injuries [4]. Cytokine storm, or cytokine storm syndrome, is caused by excessive circulating serum cytokines after inhalation injury and is also a significant complication. Progressive, widespread, systemic inflammation leads to low vascular permeability that is manifested as vasodilatory shock and progressive organ failure
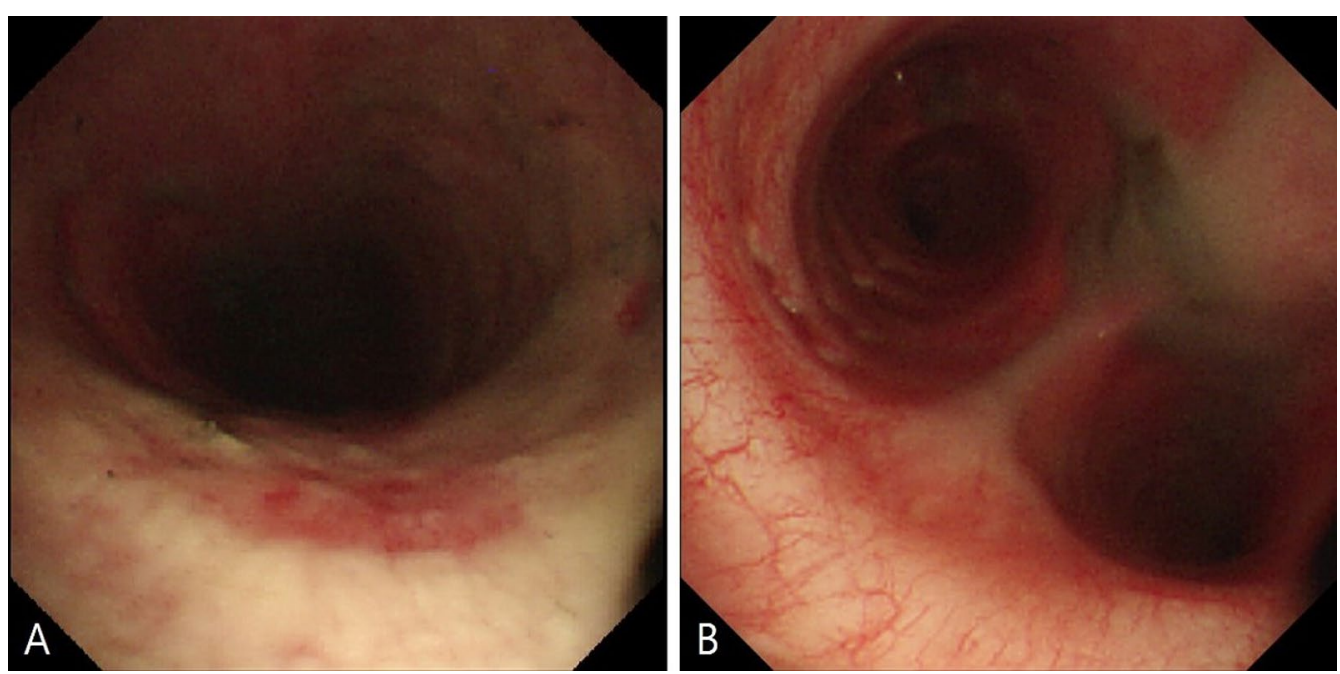

Fig. 2 Bronchoscopic findings. A Carbon soot disposition with edematous mucosal wall in upper trachea. B Hyperemic change with edema and carbon soot disposition in mucosal wall of carina and both main bronchi 

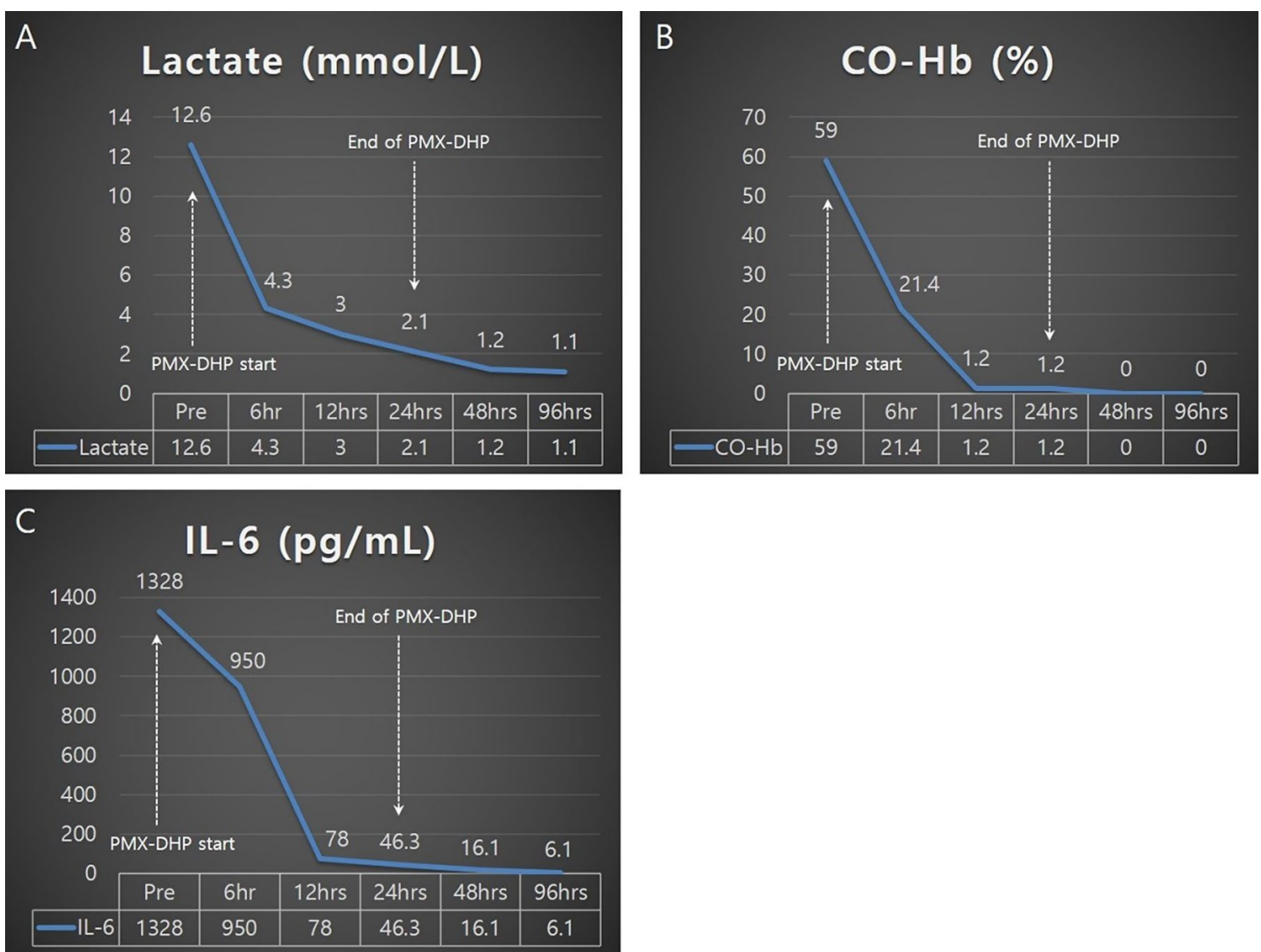

Fig. 3 Comparison of serum level of lactate (A), carboxyhemoglobin (CO-Hb) (B), and interleukin-6 (IL-6) (C) after PMX-DHP application

resulting in a poor prognosis [9]. PMX-DHP was originally developed to adsorb endotoxins released by Gramnegative bacteria during septic shock [10]. However, several studies recently reported that PMX-DHP might be useful for ARDS patients and for removal of cytokines [11-13]. Therefore, in patients with severe refractory ARDS and cytokine storm, early implication of PMXDHP for removal of cytokines and improvement of oxygenation might be considered in addition to ECMO.

\section{Conclusion}

We strongly suggest that early initiation of ECMO along with PMX-DHP even at the ED should be considered as a treatment option for severe ARDS with cytokine storm following inhalation injury to prevent mortality and to promote a better prognosis in burn patients.

\section{Abbreviations}

CO: Carbon monoxide; ARDS: Acute respiratory distress syndrome; ED: Emergency department; ECMO: Extracorporeal membrane oxygenation; PMX-DHP: Direct hemoperfusion with polymyxin B-immobilized fiber; $\mathrm{COHb}$ : Carboxyhemoglobin; IL-6: Interleukin 6; ABG: Arterial blood gas; $\mathrm{PaO2}$ : Partial pressure of oxygen; CT: Computed tomography; V-V ECMO: Veno-veno extracorporeal membranous oxygenator; CRRT: Continuous renal replacement therapy; ICU: Intensive care unit.

\section{Acknowledgements}

Not applicable.

\section{Authors' contributions}

All authors reviewed and edited the manuscript and approved the final version of the manuscript.

\section{Funding}

The authors received no financial support for the research, authorship, and/or publication of this article.

\section{Availability of data and materials}

The datasets generated during and/or analyzed during the current study are available from the corresponding author on reasonable request.

\section{Declarations}

\section{Ethics approval and consent to participate}

Haeundae Paik Hospital, Inje University College of Medicine does not require ethical approval for reporting individual cases or case series. Consent to participate was not applicable because of this article being a case report. 


\section{Consent for publication}

Written informed consent was obtained from the patient for publication of this case report and any accompanying images. A copy of the written consent is available for review by the Editor-in-Chief of this journal.

\section{Competing interests}

The authors declare that they have no competing interests

\section{Author details}

'Department of Internal Medicine, Haeundae Paik Hospital, Inje University College of Medicine, Busan 135-710, Korea. ${ }^{2}$ Department of Thoracic and Cardiovascular surgery, Haeundae Paik Hospital, Inje University College of Medicine, Busan, Korea. ${ }^{3}$ Department of Anesthesiology and Pain medicine, Haeundae Paik Hospital, Inje University College of Medicine, Busan, Korea. ${ }^{4}$ Department of Internal Medicine, Seoul ST. Mary's Hospital, The Catholic University of Korea, Seoul, Korea.

Received: 2 October 2020 Accepted: 21 July 2021

Published online: 15 September 2021

\section{References}

1. Deutsch CJ, Tan A, Smailes S, Dziewulski P. The diagnosis and management of inhalation injury: an evidence based approach. Burns. 2018;44(5):1040-51.

2. Kinoshita H, Turkan H, Vucinic S, Naqvi S, Bedair R, Rezaee R, et al. Carbon monoxide poisoning. Toxicol Rep. 2020;7:169-73.

3. Eltzschig HK, Carmeliet P. Hypoxia and inflammation. N Engl J Med. 2011;364(7):656-65.

4. Dadras M, Wagner JM, Wallner C, Huber J, Buchwald D, Strauch J, et al. Extracorporeal membrane oxygenation for acute respiratory distress syndrome in burn patients: a case series and literature update. Burns Trauma. 2019;7:28.
5. Kadri SS, Miller AC, Hohmann S, Bonne S, Nielsen C, Wells C, et al. Risk factors for in-hospital mortality in smoke inhalation-associated acute lung injury: data from 68 United States Hospitals. Chest. 2016;150(6):1260-8.

6. Enkhbaatar P, Pruitt BA Jr, Suman O, Mlcak R, Wolf SE, Sakurai H, et al. Pathophysiology, research challenges, and clinical management of smoke inhalation injury. Lancet. 2016;388(10052):1437-46.

7. Sine $C R$, Belenkiy $S M$, Buel AR, Waters JA, Lundy JB, Henderson JL, et al. Acute respiratory distress syndrome in burn patients: a comparison of the Berlin and American-European definitions. J Burn Care Res. 2016;37(5):e461-9.

8. Belenkiy SM, Buel AR, Cannon JW, Sine CR, Aden JK, Henderson JL, et al. Acute respiratory distress syndrome in wartime military burns: application of the Berlin criteria. J Trauma Acute Care Surg. 2014;76(3):821-7.

9. Clark IA, Vissel B. The meteorology of cytokine storms, and the clinical usefulness of this knowledge. Semin Immunopathol. 2017;39(5):505-16.

10. Shoji H. Extracorporeal endotoxin removal for the treatment of sepsis: endotoxin adsorption cartridge (Toraymyxin). Ther Apher Dial. 2003;7(1):108-14.

11. Tsushima K, Kubo K, Koizumi T, Yamamoto H, Fujimoto K, Hora K, et al. Direct hemoperfusion using a polymyxin B immobilized column improves acute respiratory distress syndrome. J Clin Apher. 2002;17(2):97-102.

12. Yokoyama T, Tateishi K, Tsushima K, Agatsuma T, Yamamoto H, Koizumi T, et al. A case of severe ARDS caused by novel swine-origin influenza (A) H1N1pdm) virus: a successful treatment with direct hemoperfusion with polymyxin B-immobilized fiber. J Clin Apher. 2010;25(6):350-3.

13. Ishiwari M, Togashi Y, Takoi H, Kikuchi R, Kono Y, Abe S. Polymyxin B haemoperfusion treatment for respiratory failure and hyperferritinaemia due to COVID-19. Respirol Case Rep. 2020;8(9):e00679.

\section{Publisher's Note}

Springer Nature remains neutral with regard to jurisdictional claims in published maps and institutional affiliations.
Ready to submit your research? Choose BMC and benefit from:

- fast, convenient online submission

- thorough peer review by experienced researchers in your field

- rapid publication on acceptance

- support for research data, including large and complex data types

- gold Open Access which fosters wider collaboration and increased citations

- maximum visibility for your research: over $100 \mathrm{M}$ website views per year

At BMC, research is always in progress.

Learn more biomedcentral.com/submissions 